\title{
Thermodynamics of protein folding: a random matrix formulation
}

\author{
Pragya Shukla \\ Department of Physics, Indian Institute of Technology, Kharagpur, India.
}

(Dated: October 2, 2018)

\begin{abstract}
The process of protein folding from an unfolded state to a biologically active, folded conformation is governed by many parameters e.g the sequence of amino acids, intermolecular interactions, the solvent, temperature and chaperon molecules. Our study, based on random matrix modeling of the interactions, shows however that the evolution of the statistical measures e.g Gibbs free energy, heat capacity, entropy is single parametric. The information can explain the selection of specific folding pathways from an infinite number of possible ways as well as other folding characteristics observed in computer simulation studies.
\end{abstract}

PACS numbers: $87.15 \mathrm{Cc}, 87.15 . \mathrm{hm}$ 


\section{INTRODUCTION}

The expression of a gene in a DNA leads to formation of amino acids sequences that are the basic building blocks of proteins. The message contained in a DNA then manifest through a specific structure of protein which in turn determines its functionality. In fact, the protein after its birth, acts as a feedback and leads to creation of new copies of the parent DNA.

The structure of a protein is determined purely by the amino acid sequences and its function depends on the ability of the protein to fold rapidly to its native structure [1, 2]. Based on numerous simulation studies of protein sequences (for example, see [1 11]), the folding process is believed to reveal two main characteristics: (1) a single thermodynamically stable, minimum free energy state, (2) a very short time-scale for folding e.g. milliseconds to seconds. In past, there have been several analytical attempts to explain these observations (see for example [7, 12 16]). However a thorough understanding of the rapid and selective approach of a sequence to fold to a pre-determined configuration, despite availability of an infinite number of possibilities, is still missing (referred as protein folding problem). The three main components of the missing information are: (i) an understanding of the interatomic forces which lead to native state from an unfolded state, (ii) prediction of native structure from its amino acid sequences (usually requires a prior knowledge of inter-atomic forces), (iii) the origin/ reason of fast folding speed. We seek the information by a new analytical method based on the random matrix modeling [17] of the interactions within protein as well as with its environment, and attempt to justify the findings of the simulation studies.

The interactions among various units of a biological system are often complicated and can not be determined exactly. The complexity of the interactions manifests itself through sample to sample fluctuations of the properties. Such fluctuations (different from thermodynamic or statistical ones) have been observed in a wide range of complex systems and a useful information can be extracted only from the statistical analysis of properties [17]. For

example, the microscopic energy states of complex systems like proteins are not well-defined and can at best be described by a statistical distribution. Previous analytical studies at- 
tempted to circumvent this difficulty by averaging over the ensemble of protein sequences and, therefore, could not provide information about the role of a specific sequence [7] on the folding. Our approach however is based on the averaging over the ensemble of interactions of a given sequence and does not suffer from this drawback. We analyze the interaction matrix i.e the matrix with its entries as the pairwise interactions between residues as well as their side-chains of a given sequence. The deterministic inaccuracy associated with the interactions results in their distribution (spread about some average value), with nature and degree of randomness governed by the local environment [18]. The interaction matrix then turns out to be a random matrix i.e a matrix with some/all random entries. The physical properties of such a matrix can be analyzed through their ensemble.

The concept of randomization of local interactions is essentially same in spirit as the idea of randomization of microscopic energy states, used in well-known random energy model of disordered systems [19]. The details and the information contained in random matrix model however is significantly different from that of the random energy model. The latter directly assumes a microscopic energy state to be Gaussian distributed, with all the systemspecific information contained in its mean and variance. But the explicit dependence of mean and variance on the system parameters, e.g. pairwise interaction strengths, is not known which reduces the applicability of the model in probing the folding problem. Further, the assumption of randomness in this case requires presence of disorder. In contrast, the random matrix model, based on the inaccuracy led randomization of local interactions, depends on many parameters, each being a measure of local interaction-accuracy which in turn is sensitive to the system conditions. This leads to a multi-parametric distribution of the microscopic energy states which allows one to explore the effect of local variations on the sequence. Although our analysis finally leads to a single parametric formulation of the energy states, the parameter is a well-defined functional of the system conditions. This makes the model more appropriate for the analysis of various folding stages (each described by a set of the system parameters).

A protein in aqueous solution is in equilibrium between its native (folded) and denatured (unfolded) conformations. The thermodynamic stability of the native state is based on 
the magnitude of the Gibbs free energy $G$ of the system relative to unfolded state. A negative $\Delta G=G_{f}-G_{u}$ (subscripts $f, u$ implying folded and unfolded state) indicates the native state is more stable than the denatured one. Many factors are responsible for the folding and stability of native proteins, e.g. hydrophobic interactions, hydrogen bonding, Van Der Waals forces and electrostatic interactions, conformational entropy, and the physical environment ( $\mathrm{pH}$, buffer, ionic strength, excipients etc.) [20 22]. The factors stabilizing the folded state are present in the unfolded state too and help in its stability. The folded state is however marginally more stable than the unfolded state due to various compensating factors enhancing its stability. Further the functionality and folding speed (to native conformation), instead of the thermodynamic stability, seem to be the main criteria for the selection of a natural protein conformation. Both of these characteristics require some degree of flexibility which in turn affects the free energy constraints on unfolding and refolding. These insights in the folding process are mostly based on computer simulation studies and it is desirable to seek an analytical understanding which could then help e.g in designing proteins. This motivates us to consider the partition function of a protein sequence which can be used to determine the stability measure i.e Gibbs free energy of the sequence in a specific conformation as well as the heat capacity and entropy of unfolding.

During past few decades, the attempts to explain folding and organization of proteins from the unfolded or random coil state to the native folded state have put forward many ideas. It is now believed that the polarity of proteins and their hydrophobic interaction with the solvent dominate the folding process. The hydrophilic nature of polar amino acids in aqueous solution attracts polar water molecules while non-polar amino acids tend to be hydrophobic and prefer binding with each other. These tendencies along with other factors confine the space of available conformations and the folding occurs only through specific pathways. It appears to proceed from a restricted conformation ensemble by condensation and secondary structure formation through an even smaller ensemble of "molten globules" to a well-defined, three dimensional single structure [1, 2]. The final stages of folding are also believed to depend on the specific sequence of amino acids, whereas earlier stages should be mostly insensitive to the sequence-details. Further, molecules of the same protein can 
follow different pathways to reach native state however the thermodynamic stability criteria (requiring decrease of free energy) restricts the allowed pathways. To understand these pathways, it is necessary to know the effect of varying residue-residue interactions as well as protein-solvent interactions on the thermodynamic properties. For this purpose, we analyze the energy distribution of a protein sequence under varying system conditions which leads to system-dependent formulation of thermodynamic measures.

The paper is organized as follows. The section 2 describes the energy formulation for a microscopic state corresponding to a specific conformation. The random matrix model of the interactions, based on maximum entropy principle [23], is discussed in section 3 which is used in section 4 to obtain the energy landscape i.e the distribution of a microscopic state as a function of system and environmental conditions. This information is applied in section 5 to derive the partition function and Gibbs free energy for folding. The heat capacity for denaturation and thermodynamic entropy are discussed in section 6 . The section 7 contains concluding remarks.

\section{MICROSCOPIC ENERGY STATES OF A PROTEIN SEQUENCE}

A physicist's approach to folding problem is based on applying statistical energy functions to explore a large set of alternative structures of a target protein, with native state given by the lowest energy structure. An accurate description of the Gibb's free energy function needs to take into account the many body interactions among residues, (Hydrogen bonds, ion pairs, van Der Waals interactions, hydrophobic interactions) as well as effect of the solvent. Fortunately, however, a simplified version of energy function based on pairwise contact approximation has turned out to be quite a good description in many folding simulation studies [24, 25]. Within this approximation, the energy of a particular conformation of a protein sequence of $N$ residues can be expressed in terms of a $N \times N$ contact map matrix $C$ whose matrix elements represent the pairwise contact potential: Consider a sequence $A=\left(A_{1}, A_{2}, A_{3} \ldots A_{N}\right)$, with $A_{k}$ as the amino acid at the $k^{\text {th }}$ position in the chain, folds into 
a structures whose contact map is $C$. The energy of the conformation can be given as

$$
E(C, A, U)=\sum_{k l} C_{k l} U_{k l}\left(A_{k}, A_{l}\right)=\operatorname{Tr}[C . U]
$$

with $A_{k}$ as the amino acid at the $k^{t h}$ position in the chain. Here $U$ is a $N \times N$ symmetric matrix with its elements $U_{k l}=U\left(A_{k}, A_{l}\right)$ as the interaction between residues $A_{k}$ and $A_{l}$ (present at position $k$ and $l$ of the sequence), where $A_{k}, A_{l}$ belong to a set of the twenty types of amino acids.

The contact matrix $C$ contains information about the connectedness of the sequence. Based on the connectivity between two residues, the elements of the contact matrix are usually allowed to take binary values:

$$
\begin{aligned}
C_{i j} & =1 & & \text { if residues } \mathrm{k} \text { and } \mathrm{l} \text { are connected } \\
& =0 & & \text { otherwise }
\end{aligned}
$$

The criteria for connectedness is usually considered to be the distance of the heavy atoms in the two residues: two residues are assumed to be in contact if any two heavy atoms belonging to them are closer than a threshold distance ( $1-10$ Angstroms).

The effective energy can be rewritten as

$$
E(C, A, U)=\operatorname{Tr}[H]
$$

where matrix $H$ is the product of contact matrix $C$ and interaction matrix $U$ :

$$
H_{k l}=\sum_{j=1}^{N} C_{k j} U_{j l}
$$

Eq.(3) can be applied to derive $P(E, C, u)$, the distribution of energy state $E$ for a specific $C$ matrix, or, the energy landscape for each state of protein e.g neutral, charged, folded, intermediate or unfolded (the energy of a protein being a function of the topological arrangement of the atoms) [20]. An energy landscape depicts energy as a function of the conformation for a given state of protein. The stable conformation corresponds to the global 
minimum of the landscape, with its smooth, well-correlated structure indicating the stability of the protein [20].

The energy function given in eq.(3) is one of the most studied forms in computer simulation studies of protein folding. Although this function is good enough for threading set simulations, it is believed to be not accurate enough to allow off-lattice folding simulations [26, 27]. This motivated considerations of new energy functions e.g. THOM2 which captures the environment of each residue by assigning a potential energy $U\left(A_{l}, S_{l \alpha}\right)$ for each contact $S_{l \alpha}$ to a residue $A_{l}[28]$. The total energy of a protein in this case is a sum of the site contributions:

$$
E(A, U)=\sum_{l=1}^{N} \sum_{l_{\alpha}=1}^{m_{l}} U_{l_{\alpha}}\left(A_{l}, A_{l_{\alpha}}\right)
$$

where $l=1 \rightarrow N$ with $N$ as the total number of residue sites in the sequence, $A_{l_{\alpha}}$ as the $\alpha^{\text {th }}$ contact to the residue at site $l$, with $l_{m}$ as the total number of contacts to the site 1 .

The interactions between the side chains of various residues is very crucial to achieve the 3dimensional structure of unique folded conformation. Such interactions are not taken into account in eq.(5). This motivates us to consider a generalization of eq.(5). Let $U_{l_{\alpha}, k_{\alpha}}\left(A_{l_{\alpha}}, A_{k_{\alpha}}\right)$ be the interaction strength between side chains $A_{l_{\alpha}}$ and $A_{k_{\alpha}}$, the total energy of pairwise interactions is then

$$
E(A, U)=\sum_{k, l} \sum_{\alpha=1}^{m+1} U_{k_{\alpha} l_{\alpha}}\left(A_{k_{\alpha}}, A_{l_{\alpha}}\right)
$$

Note, here the interaction between the residues is included in the sum by treating each residue as a side chain too. Due to side chain interactions, the size of the $U$-matrix is now increased: $N_{u}=\prod_{l=1}^{N}\left(l_{m}+1\right)$. The missing/ weak connections among the side-chains of different residues, and mutually dependent pairwise interactions within a single side chain, may lead to an effectively sparse form of $U$ matrix with many correlated elements.

To proceed further, we need the information about the interactions among residues in the sequence as well as with solvent. In protein simulation studies, the information is usually taken from protein data bank. However, as discussed in the next section, the PDB 
information is only approximately accurate and can be improved by taking the error into account i.e by considering the distribution of interaction strengths. The latter is then used to determine the distribution $P(E)$ and the partition function.

\section{DISTRIBUTION OF INTERACTION STRENGTHS: A RANDOM MATRIX MODEL}

Consider the interaction matrix $U$ of a protein sequence with $N$ residues with its elements $U_{k l}$ describing the pairwise interaction between residues for a given set of system conditions. For notational simplification, henceforth, we denote $U_{k l}$ by $U_{\mu}$ with $\mu \equiv\{k l\}$ as a single index (unless details required) which can take value from $1 \rightarrow M$. Here $M$ is the total number of the distinct matrix elements: $M=N(N+1) / 2$.

The presence of environment adds to the degree of complexity of the interactions in the chain. This renders an exact determination of $U_{\mu}$ technically difficult and they can be determined only within a certain degree of accuracy which, being sensitive to local system conditions, varies from element to element. Each $U_{\mu}$ can then be best described by a distribution with parameters sensitive to system conditions (see [18]).

Based on extent of available information about system conditions, the distribution of each $U_{\mu}$ can be obtained by invoking maximum entropy hypothesis [23]: in absence of any further information, the simplest and least biased hypotheses is that the system is described by the distribution $\rho(U)$ that maximizes Shannon's information entropy $S$ where

$$
S[\rho(U)]=-\int \rho(U) \ln \rho(U) \mathrm{d} \Gamma
$$

with $\Gamma(U)$ as the invariant measure in the $U$-space. For example, consider the system subjected to following constraints: (i) the probability density $\rho(U)$ is conserved (normalized to unity), (ii) each $U_{\mu}$ is described by an independent, random distribution with its higher $\operatorname{order}(>2)$ moments negligible, (iii) the mean $<U_{\mu}>=u_{\mu}$ and $2^{n d}$ moment $<U_{\mu}^{2}>=v_{\mu}^{2}+u_{\mu}^{2}$ are given by the system conditions. The maximization of Shannon entropy under these constraints leads to a Gaussian distribution of $U_{\mu}$ : 


$$
\rho(U)=\prod_{\mu=1}^{M} \frac{1}{\sqrt{2 \pi v_{\mu}^{2}}} \mathrm{e}^{-\frac{\left(U_{\mu}-u_{\mu}\right)^{2}}{2 v_{\mu}^{2}}}
$$

where $u_{\mu}$, the ensemble averaged value of interaction, could be taken e.g. from a protein data bank. Note here assumed randomness of an interaction is different from considering a "random" sequence. The components of a sequence may be well-defined but their interactions may not be.

The consideration of more realistic conditions e.g. many body interactions would introduce non-zero correlations among $U_{\mu} \mathrm{s}$ :

$$
\rho(U, v)=\tilde{C} \prod_{\mu_{1}, \mu_{2}} \exp \left[-v_{\mu_{1}, \mu_{2}}\left(U_{\mu_{1}}-u_{\mu_{1}}\right)\left(U_{\mu_{2}}-u_{\mu_{2}}\right)\right]
$$

with $v_{\mu_{1}, \mu_{2}}$ as the measures of correlations between $U_{\mu_{2}}$ and $U_{\mu_{1}}$. However in present study we confine our analysis to the independent case.

The Gaussian distributed $U_{\mu}$ (eq.(8) ) leads to a Gaussian ensemble of $H$-matrix (from eq.(41)):

$$
\rho_{H}(H, C, u)=\prod_{\mu=1}^{M} \frac{1}{\sqrt{2 \pi \nu_{\mu}^{2}}} \mathrm{e}^{-\frac{\left(H_{\mu}-b_{\mu}\right)^{2}}{2 \nu_{\mu}^{2}}}
$$

with

$$
\begin{aligned}
b_{\mu} & \equiv\left\langle H_{\mu}\right\rangle=\sum_{j} C_{k j} u_{j l} \\
\nu_{\mu} & \equiv\left\langle H_{\mu}^{2}\right\rangle-\left\langle H_{\mu}\right\rangle^{2}=\sum_{j} C_{k j}^{2}\left(u_{j l}^{2}+v_{j l}^{2}\right)-b_{\mu}^{2}
\end{aligned}
$$

As clear, $\rho_{H}$ contains sequences with different interaction energies for a given contact map as well as sequences with different contact maps for a given interaction matrix $U$.

The energy function in eq.(3) being widely used in simulation studies, it is relevant to consider the energy distribution of a sequence modeled by the ensemble $\rho_{H}$ :

$$
P(E, C, u)=\int \delta(E-\operatorname{Tr}[H]) \rho_{H}(H) \mathrm{d} H
$$

$P(E, C, u)$ contains information about the energy landscape: the existence of a clear global minimum of $P(E, C, u)$ in $C$-space for a fixed $u$ (i.e a given protein sequence) indicates its 
foldability, with the neighborhood containing information about the low-energy alternative conformations. Note the above formulation also allows the possibility to consider a more generalized form of contact matrix.

Eq.(6) being closer to realistic proteins, our main interest is to find $P(E)$ for this case:

$$
P(E, u, v)=\int \delta\left(E-\sum_{\mu} U_{\mu}\right) \rho(U, u, v) \mathrm{d} U
$$

with $\left.\mu \equiv\left\{k_{\alpha}, l_{\alpha}\right\}, \sum_{\mu} U_{\mu} \equiv \sum_{k, l, \alpha} U_{k_{\alpha}, l_{\alpha}}\right)$ and $\rho(U, v, u)$ as the density of the ensemble of $U$-matrices, each of size $N_{u}$. Assuming the matrix elements correlations negligible, it can again be described by eq.(8) with now $M=N_{u}\left(N_{u}+1\right) / 2$.

Eq.(13) can model various protein states e.g folded or unfolded. For example, the interactions between side chains in an unfolded state is much weaker in comparison to a folded state. The unfolded state can be described by eq.(8) by taking $u_{\mu} \rightarrow 0, v_{\mu} \rightarrow 0$ if $\mu \equiv\left\{k_{\alpha}, l_{\alpha}\right\}$ is such that $k \neq l$ and if $A_{k_{\alpha}}$ and $A_{l_{\alpha}}$ correspond to the side chains. For native state, a well-defined three-dimensional structure, a large number of $u_{\mu}$ s would be non-zero with corresponding $v_{\mu}$ very small. Similarly the intermediate folding states would correspond to varying $\left(u_{\mu}, v_{\mu}\right)$-strengths, based on the sequence and its environment. The transition from unfolded to folded state can therefore be studied by a variation of these parameters.

\section{EVOLUTION OF P(E) DURING FOLDING PROCESS}

Let us first consider the $P(E)$ given by eq.(13) .

As the folding proceeds, the interaction strengths of residues with each other as well as with local environment change and the residues in the sequence rearrange themselves (dictated by their chemical nature and affinities). The folding therefore corresponds to dynamics of the elements $U_{\mu}$ and an evolution of $\rho(U)$ in the $U$-matrix space.

The deterministic accuracy of each $U_{\mu}$ also fluctuates rapidly as the folding evolves, with different "time-scale" of fluctuations for each matrix element. This corresponds to a change of distribution parameters of the ensemble of the interaction strengths of a given sequence. The folding process can then be considered as an evolution of the ensemble in the parametric space. Both describing the same process, the parametric space dynamics of 
$\rho(U, u, v)$ is therefore expected to mirror itself in its $U$-space dynamics. This is indeed the case as can be seen by a partial differentiation of eq.(8) with respect to $\left(u_{\mu}, v_{\mu}\right)$; a specific combination of the first order parametric variations turns out to be equivalent to a diffusion dynamics of $U_{\mu}$ along with a drift component:

$$
-\gamma\left[2 x_{\mu} \frac{\partial \rho}{\partial x_{\mu}}+b_{\mu} \frac{\partial \rho}{\partial b_{\mu}}\right]=\frac{\partial}{\partial U_{\mu}}\left[\frac{g_{\mu}}{2} \frac{\partial}{\partial U_{\mu}}+\gamma U_{\mu}\right] \rho
$$

where $x_{\mu} \equiv 1-\left(2-\delta_{\mu}\right) v_{\mu}, g_{\mu} \equiv g_{k l}=1+\delta_{k l}$ with $\delta_{k l}=1$ for $k=l$ and 0 for $k \neq l$.

Multiplication of both sides of eq.(14) with factor $\delta\left(E-\sum U_{\mu}\right)$ and subsequent integration over $U$-space gives, along with eq.(13),

$$
\gamma \sum_{\mu=1}^{M_{0}} \frac{\partial P}{\partial z_{\mu}}=\frac{\partial}{\partial E}\left[\frac{\partial}{\partial E}+\gamma E\right] P
$$

with $z_{\mu}=-\frac{1}{2} \ln \left(\left|x_{\mu}\right|\left|b_{\mu}\right|^{2}\right), M_{0}$ as the number of non-zero parameters $x_{\mu}, b_{\mu}$ and $\gamma$ as an arbitrary constant with units of $E^{-1}$.

As eq.(15) indicates, the combined effect of first order parametric variations is a diffusion of $P(E)$ in the energy space. Due to linearity, these first order changes are additive in nature. The collective response of the sequence to these changes can then be mimicked by the response to a single parameter $Y$ :

$$
\frac{\partial P}{\partial Y}=\frac{\partial}{\partial E}\left[\frac{\partial}{\partial E}+\gamma E\right] P
$$

where $Y$ is defined by the condition $\frac{\partial P}{\partial Y}=\sum_{\mu=1}^{M_{0}} \frac{\partial P}{\partial z_{\mu}}$ or, alternatively,

$$
\sum_{\mu=1}^{M_{0}} \frac{\partial Y}{\partial z_{\mu}}=1
$$

The above condition can easily be solved to give

$$
Y=\frac{1}{\gamma M_{0}} \sum_{\mu=1}^{M} a_{\mu} z_{\mu}+c_{0}
$$

with $M_{0}=\sum_{\mu} a_{\mu}$ and $c_{0}$ is a constant determined by the initial condition. Here $a_{\mu}$ are arbitrary constants which can be fixed by physical considerations as follows. Eq.(18) describes 
$Y$ as a a weighted average of $z_{\mu} \mathrm{s}$, each representing local accuracy fluctuations. Assuming no particular bias of folding to any specific error, all $a_{\mu} \mathrm{s}$ can be chosen equal. This gives

$$
Y=-\frac{1}{2 \gamma M_{0}} \prod_{\mu}^{\prime} \ln \left[\left|x_{\mu}\right|\left|b_{\mu}\right|^{2}\right]+c_{0}
$$

here $\prod^{\prime}$ implies a product over non-zero $b_{\mu}$ and $x_{\mu}$ and $c_{0}$ is a constant determined by the initial condition (i.e unfolded sequence). (A mathematically rigorous derivation of $Y$ can be found in [18, 29]). Being a function of the system conditions governing folding e.g. interaction strengths as well as local environment, $Y$ can be termed as the folding parameter. During folding, therefore, $P$ undergoes a $Y$-governed diffusion due to accuracy driven random forces, along with a finite drift caused by external forces e.g. environmental conditions.

Eq.(16) describes the flow of the probability $P\left(E, Y \mid E_{0}, Y_{0}\right)$ from an arbitrary initial ensemble of the matrices $H_{0}$ to a steady state (occurring in the limit $\frac{\partial \rho}{\partial Y} \rightarrow 0$ ); the steady state turns out to be a Gaussian free of any system conditions: $P(E, Y \rightarrow \infty) \propto \mathrm{e}^{-\gamma E^{2} / 2}$. For an arbitrary initial state $P\left(E_{0}, Y_{0}\right)$, eq.(16) can be solved to give

$$
P\left(E, Y \mid E_{0}, Y_{0}\right)=c \exp \left[-a\left(E-\alpha E_{0}\right)^{2}\right]
$$

with $a=\frac{1}{2\left(1-\alpha^{2}\right)}, c=\frac{1}{\sqrt{1-\alpha^{2}}}$, and $\alpha=\mathrm{e}^{-\left(Y-Y_{0}\right)}$. Let $P\left(E_{0}, Y_{0}\right)$ represents the energy landscape of the denaturated state. The probability $P\left(E, Y-Y_{0}\right)$ for various intermediate stages between denaturated and native state can then be obtained by integrating eq.(20) over $P\left(E_{0}, Y_{0}\right)$ :

$$
P\left(E ; Y-Y_{0}\right)=\int P\left(E, Y \mid E_{0}, Y_{0}\right) P\left(E_{0}, Y_{0}\right) \mathrm{d} E_{0}
$$

As eq.(19) indicates, $Y$ increases as folding proceeds; this is due to increasing contributions from non-zero $u, v$ parameters.

$P\left(E, Y-Y_{0}\right)$, given by eq.(21), describes the energy landscape for a specific folding stage represented by the functional $Y(u, v)$ which contains information about the system conditions prevailing during that stage. Thus, beginning from an unfolded sequence, the folding 
process is governed by the collective influence (described by $Y$ ) of the local interactions (among residues as well as environment) on the protein dynamics. Different alternatives for pairwise interactions may result in different $Y$ functions and therefore many trajectories originating from a given unfolded state. The thermodynamic conditions however restrict the choice of the folding trajectories. As discussed in the next section, $Y$ dependence of $P\left(E, Y-Y_{0}\right)$ leads to $Y$-governed evolution of the thermodynamic measures e.g Gibbs free energy $G$ during folding. The thermodynamic stability criterion restricts the native state to occur along the trajectory with a well-defined global minimum of $G$ occurring, say at $Y=Y_{F}$. Due to its dependence on the value of $Y$ and not on its functional form, $G(Y)$ may take a same value at more than one trajectory. Thus folding occurs along trajectories with an approximately similar $G(Y)$ behavior, leading to a common global minimum, say at $Y=Y_{F}$. Existence of a local minimum for $Y<Y_{F}$ may inhibit the folding to a native state. Similarly a local minimum occurring for $Y>Y_{F}$ may lead to misfolding with changing environmental conditions.

As eq.(21) shows, different energy landscapes of initial sequences may lead to different native states. However if mutations of some of the residues leave $P\left(E_{0}, Y_{0}\right)$ of an unfolded sequence unchanged, the native state then will also remain unaffected; this is in agreement with observed robustness of the native state to sequence-mutations.

The initial ensemble, that is, the ensemble of unfolded or fully denatured protein is a linear sequence of residues with no secondary of tertiary structure, often existing as a random coil where all conformations have comparable energies. $P\left(E_{0}, Y_{0}\right)$ in this case can be described by a Gaussian distribution:

$$
P\left(E_{0}, Y_{0}\right)=\frac{1}{\sqrt{2 \pi \eta}} \mathrm{e}^{-\frac{\left(E_{0}-\epsilon\right)^{2}}{2 \eta}}
$$

As clear from their functional forms, eq.(14) is also valid for $\rho_{H}(H)$ (eq.(10)), after replacing $v_{\mu} \rightarrow \nu_{\mu}, b_{\mu} \rightarrow u_{\mu}, \sum_{k, l, \alpha} \rightarrow \sum_{k, l}$ and $\rho(U) \rightarrow \rho_{H}(H)$. Consequently, eq.(16) describes the the evolution of $P(E)$, given by eq.(12), too, with corresponding changes in $Y$. 


\section{PARTITION FUNCTION AND GIBBS FREE ENERGY $G$}

Eq.(21) for $P\left(E ; Y-Y_{0}\right)$ can now be used to obtain the partition function $Z$ for the conformation ensemble described by the complexity parameter $Y$ :

$$
\begin{aligned}
Z\left(Y-Y_{0}, T\right) & =\int \mathrm{e}^{-\beta E} P\left(E, Y-Y_{0}\right) \mathrm{d} E \\
& =\sqrt{\frac{\pi}{2 a^{2}}} \mathrm{e}^{\frac{\beta^{2}}{4 a}} Z_{0}\left(Y_{0}, \tau\right)
\end{aligned}
$$

where $\tau \equiv T / \alpha, \beta=1 / k T$ and

$$
Z_{0}\left(Y_{0}, \tau\right)=\int \mathrm{e}^{-\alpha \beta E_{0}} P\left(E_{0}, Y_{0}\right) \mathrm{d} E_{0}
$$

The free energy of the conformation at temperature $T$ is then given by

$$
\begin{aligned}
G\left(Y-Y_{0}, T\right) & =-k T \ln Z \\
& =\alpha G_{0}(\tau)-\frac{1-\alpha^{2}}{2 k T}-k T \ln (\sqrt{2 \pi})
\end{aligned}
$$

where $G_{0}(\tau)$ is the free energy of the unfolded state at rescaled temperature $\tau$ :

$$
G_{0}(\tau)=-k \tau \ln Z_{0}
$$

As eq.(26) implies, the evolution of $G$ at a given temperature $T$ is dictated by $\alpha$, and therefore, $Y$, a function of system conditions through $\left\{u_{\mu}\right\}$ and $\left\{v_{\mu}\right\}$. The influence of system conditions on $G$ can then be studied through $Y$.

The stability of a conformation increases as its $G$ decreases relative to that of the unfolded protein. The thermodynamic stability criterion for folded conformation requires its free energy to be minimum. This can be achieved by seeking system conditions i.e $\alpha=\alpha_{f}$ at fixed $T$ for which

$$
\left.\frac{\partial G}{\partial \alpha}\right|_{\alpha_{f}, T}=0,\left.\quad \frac{\partial^{2} G}{\partial \alpha^{2}}\right|_{\alpha_{f}, T}<0
$$

or equivalently,

$$
\beta \alpha_{f}+G_{0}\left(\beta \alpha_{f}\right)+\left.\alpha \frac{\partial G_{0}\left(\beta \alpha_{f}\right)}{\partial \alpha_{f}}\right|_{T}=0 .
$$


and

$$
\left.\alpha_{f}^{2} \frac{\partial G_{0}^{2}}{\partial \alpha_{f}^{2}}\right|_{T}-\beta \alpha_{f}-2 G_{0}\left(\beta \alpha_{f}\right)<0
$$

Substitution of $G_{0}$ in eq.(29) leads to complexity parameter for the thermodynamically stable conformation at a fixed temperature $T: Y_{f}=Y_{0}-\ln \left(\alpha_{f}\right)$.

For example, for an unfolded sequence given by eq.(22), eq.(25) and eq.(27) give

$$
Z_{0}(\tau)=\mathrm{e}^{\frac{-(2 \epsilon k \tau-\eta)}{2 k^{2} \tau^{2}}}, \quad G_{0}(\tau)=\epsilon-\frac{\eta}{2 k \tau}
$$

This on substitution in eqs.(29, 30) gives

$$
\alpha_{f}=\mathrm{e}^{-\left(Y_{f}-Y_{0}\right)}=\frac{\epsilon}{(\eta-1) \beta},\left.\quad \frac{\partial G^{2}}{\partial \alpha_{f}^{2}}\right|_{T}=(1-\eta) \beta .
$$

The native state can therefore occur only if $\epsilon>0, \eta>1$.

As eq.(26) implies, the stability of a given conformation changes with temperature too. The temperature $T_{m}$ for maximum stability of a given conformation, with all other system conditions fixed, can be obtained by the condition $\left.\frac{\partial G}{\partial T}\right|_{\alpha}=0$ which gives

$$
\left.2 \frac{\partial G_{0}(\tau)}{\partial \tau}\right|_{\alpha}+k \beta^{2}\left(1-\alpha^{2}\right)-2 k \log (\sqrt{2 \pi})=0
$$

or, alternatively, with $S_{0}(\tau)=-\left.\frac{\partial G_{0}(\tau)}{\partial \tau}\right|_{\alpha}($ entropy at a fixed $\alpha)$,

$$
2 S_{0}(\tau)-k \beta^{2}\left(1-\alpha^{2}\right)+2 k \log (\sqrt{2 \pi})=0
$$

By substituting $S_{0}$ in the above equation, one can determine $T_{m}$ for a specific $\alpha$ i.e a sequence in a specific solvent. As eq.(34) suggests, the stability of a structure decreases if the temperature $T>T_{m}$ or $T<T_{m}$; this also agrees with the simulation studies. For example, for $P\left(E_{0}, Y_{0}\right)$ given by eq.(22),$\left.S_{0}(\tau)\right|_{\alpha}=-\frac{\eta}{2 k \tau^{2}}$. Eq.(34) then gives $T_{m}=\frac{\left(1-\alpha^{2}\right)+\eta \alpha^{2}}{k^{2} \ln (2 \pi)}$. It is easy to check that $\left.\frac{\partial^{2} G}{\partial T^{2}}\right|_{\alpha}<0$ at $T=T_{m}$, indicating a decreasing $G(T)$ and therefore stability for $T>T_{m}$ or $T<T_{m}$.

Note $\alpha$ (through $Y$ ) depends on both, interactions within sequence as well as with the environment (through set $\{u, v\}$ ). Following our approach, the folding therefore occurs when the matrix $v$ (or $C$ for case eq.(12)), for a specific interaction matrix $u$, will satisfy 
eq.(29) with $G_{0}$ of the unfolded state at a temperature $T / \alpha$. The approach also explains the existence of specific folding pathways at a fixed temperature: $Y_{F}$ in the parametric space $\left\{u_{\mu}, v_{\mu}\right\}$ is connected to $Y_{0}$ through several paths however folding occurs along paths with relatively maximum stability (among all paths) for an intermediate state too. These folding paths correspond to minimum free energy change between any two intermediate points. Also note that $Y$ (through $\{u, v\}$ ) is evolving with time the rapidity of which depends on the environment; the information may help in the determination of folding speed at a fixed temperature.

The appearance of $G_{0}$ in eqs.(26+34) indicates that the information specifying the native structure as well as the pathway to attain that state is contained in the amino acid sequence of each protein. The presence of both $\alpha$ and $T$ in these equations however reveals the dependence of folding process on environmental factors as well as various interactions among residues. Thus nearly identical amino acid sequences may not fold similarly if their environment is different. This is in agreement with the results obtained by simulation studies of proteins.

\section{HEAT CAPACITY AND ENTROPY FOR DENATURATION}

Heat capacity $C_{p}$ (at constant pressure p), defined as

$$
C_{p}=\left.\frac{\partial\langle E\rangle}{\partial T}\right|_{p}=\left.k \beta^{2} \frac{\partial^{2}(\beta G)}{\partial \beta^{2}}\right|_{p}
$$

is an important measure to study the dynamics of unfolding [21] and the hydrophobic effect on the protein stability.

As eq.(29) and eq.(34) indicate, the existence of a thermally stable native state depends on the specific relation of $G_{0}, Y$ and $T$. It may not be satisfied by a sequence under certain environmental conditions; the protein then will not fold into its biochemically functional form. Further a folded conformation may unfold or "denature" if changes in system conditions e.g temperatures, concentrations of solutes, $\mathrm{pH}$ conditions, mechanical forces, and the chemical denaturants result in violation of the eq.(29) or eq.(34). The effect of all these changes on $C_{p}$ can be studied through its $Y$-formulation (obtained from eq.(35) and eq.(26) ): 


$$
\begin{aligned}
C_{p}(\tau) & =\frac{k \beta^{2}}{\alpha}\left[\left.\beta^{2} \alpha^{2} \frac{\partial^{2} G_{0}}{\partial \beta^{2}}\right|_{p}+\left.2 \frac{\partial G_{0}}{\partial \beta}\right|_{p}+\alpha\left(\alpha^{2}-1\right)\right] \\
& =C_{p 0}(\tau)+k \beta^{2}\left(\alpha^{2}-1\right)
\end{aligned}
$$

with $C_{p 0}(\tau)=\left.k \beta_{0}^{2} \frac{\partial^{2}\left(\beta_{0} G\right)}{\partial \beta_{0}^{2}}\right|_{p}$ as the heat capacity of unfolded protein at temperature $\tau=T / \alpha$ and $\beta_{0}=1 / k \tau$.

The unfolding primarily occurs due to exposure of side chains (e.g. non-polar groups), buried in the native state, to solvent. The folding is believed to be dominated by the polar groups binding helped by solvent. Both these process involve $C_{p}$ change; for a sequence going from a state " $Y_{i} "$ to " $Y_{f}$ " at temperature $T$, the change in specific heat $\Delta C_{p} \equiv C_{p}\left(\tau_{f}\right)-C_{p}\left(\tau_{i}\right)$ can be given as (from eq.(37)),

$$
\Delta C_{p}=C_{p 0}\left(\tau_{f}\right)-C_{p 0}\left(\tau_{i}\right)+k \beta^{2}\left(\alpha_{f}^{2}-\alpha_{i}^{2}\right)
$$

with $\alpha_{k}=\mathrm{e}^{-\left(Y_{k}-Y_{0}\right)}$ for $k=f, i$. Due to positive and negative $C_{p}$ of hydration for apolar and polar groups, respectively, the sign of $\Delta C_{p}$ can provide information about nature of solvation e.g polar or apolar, and folding/ unfolding/ misfolding etc. For example, for the unfolded state given by eq.(22),

$$
\Delta C_{p}=k \beta^{2}(1-\eta)\left(\alpha_{f}^{2}-\alpha_{i}^{2}\right)
$$

Thus for unfolding, which corresponds to $Y_{f}<Y_{i}$ or $\alpha_{f}>\alpha_{i}$, one gets $\Delta C_{p}>0$. The folding, with $Y_{f}>Y_{i}$, similarly corresponds to $\Delta C_{p}<0$.

The entropy

$$
S=k(\ln Z+\beta E)=k \beta^{2} \frac{\partial G}{\partial \beta}
$$

is another important thermodynamic property commonly measured for proteins. A competition of entropy with stabilizing forces determines the possibility of unfolding which occurs at temperatures when $S$ becomes dominant. The $Y$-dependence of $S$ can be given as

$$
S(Y, T)=S_{0}(\tau)-(1 / 2) k \beta^{2}\left(1-\alpha^{2}\right)+k \ln \sqrt{2 \pi}
$$

with $S_{0}(\tau)$ as the entropy of unfolded sequence at temperature $\tau$. 
The entropy change $\Delta S$ contains information about reversibility $(\Delta S \leq 0)$ or irreversibility $(\Delta S>0)$ of the process. $Y$ - dependence of $\Delta S$ can then be used to determine the system-condition which can lead to refolding of a misfolded protein. For example, presence of chaperon molecules may change interaction parameters (referred by $u$ ) and therefore $Y$ and $\Delta S$. For a sequence changing from state $Y_{i} \rightarrow Y_{f}, \Delta S$ is

$$
\Delta S=S_{f}-S_{i}=S_{0}\left(\tau_{f}\right)-S_{0}\left(\tau_{i}\right)+(1 / 2) k \beta^{2}\left(\alpha_{f}^{2}-\alpha_{i}^{2}\right)
$$

For unfolded state given by eq.(22), we get

$$
\Delta S=k \beta^{2}(1+\eta)\left(\alpha_{f}^{2}-\alpha_{i}^{2}\right) / 2
$$

which implies an increase of entropy for $Y_{f}<Y_{i}$ (unfolding) and a decrease of entropy for $Y_{f}>Y_{i}$ (folding).

The simulation studies suggest that the ratio of the entropy change, $\Delta S$, to the heat capacity change, $\Delta C_{p}$, for the dissolution of a variety of hydrophobic compounds is a constant. This is confirmed by our formulation too. The ratio can be determined from eq.(35) and eq.(40):

$$
\frac{\Delta S}{\Delta C_{p}}=\frac{\partial^{2}}{\partial \beta^{2}} \ln \frac{Z_{f}}{Z_{i}}
$$

It is easy to see, from eq.(39) and eq.(43), that the ratio depends only on the properties of unfolded sequence: $\frac{\Delta S}{\Delta C_{p}}=\frac{1}{2} \frac{1+\eta}{1-\eta}$.

\section{CONCLUSION}

To summarize, a protein sequence in general is described by a multi-parametric ensemble of interactions. Our study shows however that the thermodynamic properties of the sequence are governed by a single parameter (besides temperature) which is basically a measure of average uncertainty associated with the local interactions. The formulation provides an analytical understanding of some important observations obtained by computer simulation 
studies of proteins e.g dependence of native state on original sequence, the role of solvent, decrease of stability of the native state above and below the critical temperature. The stability of folded sequence against mutations can also be explained by the $Y$-dependence of free energy: a mutation may change the interaction parameter $u_{k l}$ however $Y_{F}$ may remain unaffected (change being averaged out in the combination of interaction parameters). Such mutations will leave native state unaffected. The $Y$-formulation also explains the selection of specific folding pathways among infinite number of possibilities and can be used to identify them. We have yet to apply it to many other simulation studies observations, for example, the observed preference to the functionality and folding speed, instead of stability, as the main criteria for selection of a natural protein conformation, studies on misfolding of proteins etc.

The random matrix approach described here is applicable only for the cases of interaction matrix with independent matrix elements; this takes into account only two-body interactions. In general, a protein is a complex system with many body interactions and consequently the interaction matrix contains correlated elements. The generalization of single parametric formulation to protein models with correlated matrix elements is very desirable; we intend to pursue some of these questions in future studies.

[1] Creighton, T.E., Biochem J. (1990) 270, 1-16.

[2] Richards, F.M., Scientific American (Jan 1991), 34-41.

[3] Li H, Helling R, Tang C, Wingreen N, Science 273:666669, (1996); Bloom JD, Labthavikul ST, Otey CR, Arnold FH, Proc Natl Acad Sci. USA 103:58695874, (2006); Xia Y, Levitt M, Protiens Struct Funct Bioinform 55:107114, (2004); Sun SJ, Brem R, Chan HS, Dill KA (1995) Protein Eng 8:1205, (1995); Park S, Xi Y, Saven JG, Curr Opin Struct Biol 14:487494, (2004); Kleinberg J, Proceedings of the Third Annual Association for Computing Machinery International Conference on Research in Computational Molecular Biology (ACM RECOMB), eds Istrail S, Pevzner P, Waterman M (ACM Press, New York), Vol 3, 226237, (1999).

[4] Saven J. G., Curr Opin Struct Biol 12:453458, (2002); Koehl P., Levitt M. (2002) Proc Natl 
Acad Sci USA 99:12801285, (2002). Larson S. M. , England J. L., Desjarlais J. R., Pande V. S., Protein Sci 11:2804 2813, (2002); Bradley P, Misura KMS, Baker D, Science 309:18681871, (2005).

[5] Lee S, Tsai F. J. Biochem. Mol. Biol. (2005), 38 (3): 25965.

[6] Shortle D, FASEB J. 10 (1): 2734. PMID 8566543.

[7] Hao, M.H., and Scheraga, H.A., J. Phys. Chem. (1994) 98:9882-9893.

[8] Shakhnovich E. I., Faztidinov G, Gutin A. M., and Karplus M., Phys. Rev. Lett., 1991, 67, 1665 .

[9] Shakhnovich E. I. and Gutin A. M. Proc. Natl. Acad. Sci. U.S.A., 1993, 90, 7195.

[10] Camcho C. J. and Thirumalai D, Proc. Natl. Acad. Sci. U.S.A., 1993, 90, 6369.

[11] Leopold P. E, Montal M., Onuchic J. N., Proc. Natl. Acad. Sci. U.S.A 1992, 89, 8721.

[12] T. X. Hoang, Seno F., Antonio T., Banavar J. R., Maritan A., J. Chem. Phys. 129, 035102, (2008).

[13] Seno F. et al, Phys. Rev. Lett. 100, 078102, (2008).

[14] Shakhnovich E (1998) Fold Des 3:4558, (1998); Betancourt M. R., Thirumalai D (2002) J Phys Chem 106:599609, (2002); Lau K. F., Dill K (1990) Proc Natl Acad Sci USA 87:638642, (1990); Shakhnovich E. I. (1994) Phys Rev Lett 72:39073911, (1994); Govindarajan S, Goldstein RA, Proc Natl Acad Sci USA 93:33413345, (1996).

[15] Bryngelson J. D, and Wolynes P. G, Proc. Natl. Acad. Sci. U.S.A., 1987, 84, 7524; J. Phys. Chem., 1989, 93, 6902.

[16] Shakhnovich E. I. and Gutin A. M. Biophys. Chem. 1989, 34, 187; Nature 1990 346, 773.

[17] Mehta M. L.,Random Matrices, (Academic Press, New York, 1991); Guhr T., MullerGroeling G. A. and Weidenmuller H. A., Phys. Rep. 299, 189, (1998).

[18] Shukla P., J. Phys. A: Math. Theo, 41, 304023, (2008).

[19] Derrida B., Phys. Rev. B, 24, 2613, (1981).

[20] Frauenfelder, H, and Wolynes, P.G., Physics Today (Feb 1994), 58-64.

[21] Murphy K. P., Privalov P. L., Gill S. J., Science (Feb 1990) 247, 559-561

[22] van den Berg B, Wain R, Dobson C M, Ellis R J, EMBO J. 19 (15): 38705. 
[23] Balian R., Nuovo Cimento Soc. Itul. Fis. B, 57, 183, (1968).

[24] Bastolla U., Vendruscolo M., and Knapp E., PNAS, 2000, 97, 3977.

[25] Vendruscolo M., Maritan A. and Banavar J., Phys. Rev. Lett. 1996, 78, 3967.

[26] Vendruscolo M., and Domany E., J. Phys. Chem., 1998, 109, 11101.

[27] Vendruscolo M., Najmanovich R. and Domany E., Phys. Rev. Lett., 1999, 82, 656.

[28] Meyerguz L., Kleinberg J., and Elber R., PNAS (2007), 104, 11629.

[29] Shukla, P. Phys. Rev. E, 71, 026226, (2005); Phys. Rev. E 62, 2098, (2000). 